\title{
Ferramentas alternativas para monitoramento e mapeamento automatizado do conhecimento
}

\section{Lúcia $\mathrm{C}$ unha 0 rtiz}

Cientista da Informação responsável pela Secretaria de M onitoramento de I nformação C ientífica eT ecnológica do C M D M C I C epid/F apesp - U FSC ar - D epartamento de física Grupo de Supercondutividade e Magnetismo.

lucia@df.ufscar.br.

\section{Wilson A ires 0 rtiz}

Professor adjunto do D epartamento de F ísica da U FSC ar. Vice-diretor do $C$ entro Multidisciplinar de $\mathrm{D}$ esenvolvimento de $\mathrm{M}$ ateriais C erâmicos - C M D M C / C epid/F apesp - U F SC ar - D epartamento de Física G rupo de Supercondutividade e M agnetismo wortiz@df.ufscar.br.

\section{Sergio L uisda Silva}

Professor assitente do D epartamento de C iência da Informação da UFSC ar. D outorando epesquisador do EM U M A/EESC / U SP - U FSC ar - Departamento de C iência da Informação da U FSC ar. sergiol@ power.ufscar.br.

\section{Resumo}

$A$ análise da informação é uma excelente estratégia para monitoramento, pesquisa e desenvolvimento em todos os ramos do conhecimento. O objetivo primordial deste trabalho foi consolidar um método alternativo empregando ferramentas eletrônicas na realização do monitoramento automatizado da informação e em sua análise bibliométrica. O trabalho foi desenvolvido tendo como suporte a base Web of Science, do Institute for Scientific Information (ISI), e o uso de softwar como Word, Excel, Reference Manager e Origin. A título de exemplo, aplicamos o método à área de desenvolvimento de produtos, obtendo como resultados uma lista de descritores, a relação dos periódicos mais importantes da área, os autores mais produtivos e uma indicação das parcerias mais freqüentes entre eles.

\section{Palavras-chave}

Monitoramento da informação; Biblioteconomia; Ciência da informação.

\section{Alternative tools for computer-assisted monitoring and mapping of knowledge}

\begin{abstract}
Analysis of information is an excellent strategy for Monitoring, Research and Development in all branches of knowledge. This work was developed using Web of Science's database, from ISI (Institute for Scientific Information). The main objective was to establish an alternative method employing electronic tools in computer-assisted monitoring of information and its bibliometric analysis. Also used were the software Word, Excel, Reference Manager and Origin. As an application example, the method was applied to the area of Product Development, and the output results of the research were a list of keywords, a relation of the most important journals in the area, the most productive authors, and an indication of the most frequent partnerships among them.
\end{abstract}

Keywords

Information monitoring; Bibliometry; Information science.

\author{
INT RO DUÇÃ O
}

0 desenvolvimento científico etecnológico mundial tem crescido enormemente nas últimas décadas. U ma das mais claras manifestações desse crescimento espetacular éo surgimento deum imenso número deveículos emeios de divulgação voltados àcomunidadetécnico-científica. A tualmente, além de um número muito grande de periódicos e publicações especial izadas em papel, tem ocorrido um aumento significativo nautilização demeios eletrônicos dedivulgação.

A lém disso, registre-se a crescente especial ização destes meios, que desdobram umaárea de conhecimento em várias unidades, por um lado facilitando a busca por meio da divisão, mas, ao mesmo tempo, dificultando-a pela pulverização de cada assunto em domínios específicos.

A realização de atividades de pesquisa e desenvolvimento, como aquelas executadas em laboratórios universitários, requer um trabalho sistemático de monitoramento eprospecção das áreas deconhecimento principais e correlatas às suas atividades. Estetrabal ho de busca, análisee síntese de informações emprega cada vez mais metodologias e ferramentas baseadas em bibliometria, estatística esistemas de informação, sem 0 que se tornaria praticamente impossível acompanhar a informação disponível, crescente em quantidade e diversidade, bem como sua disseminação com o auxílio dediferentes suportes.

0 uso destas metodologias eferramentas, genericamente classificado como tratamento automatizado da informação, ou bibliometria automatizada, consiste basicamenteda aplicação defiltros paraclassificar eseparar a informação coletada, bem como agregar-Ihe valor. Esta abordagem tem sido empregada para disponibilizar, por meio de indicadores de tendências, informações vitais, seja para o pesquisador ou para outros tomadores de decisão.

0 tratamento da informação é a base do processo de monitoramento tecnológico, ou, deum modo mais geral, da inteligência competitiva. 0 monitoramento tecnológico consiste em pesquisar o assunto escolhido, que pode ser um produto, uma tecnologia, uma instituição, pesquisadores, fontes de informação 
científica etecnológica, dentre outras ligadas ao assunto focalizado. A inteligência competitiva consiste na aquisição, análise, compreensão, síntese edifusão interna deinformações de diversas fontes etipos, sobretodos os assuntos de interesse da instituição em questão, visando a ajudar os tomadores de decisão ( M cG onagle, 1998).

Inteligência é a informação analisada, e inteligência competitivaé, portanto, a informação analisada para a competitividade. $\mathrm{N}$ o Brasil, a busca pelo aumento na competitividade tem despertado grande interesse por esses estudos. A bibliometria aparece no contexto da inteligência competitiva como aferramenta que el abora indicadores detendências, gráficos, figuras emapas que vão sintetizar as informações paraa tomada de decisão.

A partir do universo de metodologias e ferramentas já existentes, desenvolvemos um procedimento que permitiu criarmos uma classificação e um Mapa de C onhecimentos, que pode ser um minidicionário ou até mesmo um minitesauro da área pesquisada, com a finalidade de facilitar as buscas deinformação por parte dos pesquisadores, focando ao máximo o universo de buscae retendo apenas a parteútil das informações. Esta tarefafoi conduzidatendo-secomo fontedeinformação um conjunto mundial de periódicos indexados, disponíveis no banco de dados internacional do I nstitute for Scientific I nformation (ISI).

O procedimento desenvolvido para geração da classificação e do $\mathrm{M}$ apa deC onhecimentosfoi testado mediante aplicação na área de desenvolvimento de produtos. Segundo Toledo (1993), o desenvolvimento de produtos situa-se na interface entre a empresa e 0 mercado, daí sua importância estratégica, cabendo-Ihe chegar a um bem que atenda às expectativas do mercado eque possaser produzido deforma eficiente.

De posse de um Mapa de C onhecimentos, pode-se ter uma melhor orientação sobre os descritores - ou conheci mentos - que devem ser monitorados, bem como a intensidade, a freqüência, assim como as formas defazêlo.

\section{Tratamento da informação}

M uitas ainda são as dificuldades encontradas pararealizar o procedimento operacional da análise bibliométrica. U ma das mais críticas éafal ta de padronização entreos formatos disponíveis para recuperação dainformação nas bases de dados, chegando muitas vezes a serem incompatíveis até com os formatos exigidos pelos softwarespara tratamento de dados. A lguns dos softwares utilizados no tratamento automatizado da informação são I nfotrans, I nfobanks, I deal ist, D ataview, Patent Trend A nalysis, M atrisme, A ccess, Statística, eExcel, (Portter, 1981; Rostaing, 1996).

Segundo Sotolongo (1999), muitos pesquisadores têm buscado novas alternativas para seus estudos bibliométricos, não só pelo preço, que nem sempre é acessível a todos, mas também como umaforma de criar o pções para estudos e análises nessa área. 0 presente artigo decorre desta mesma preocupação de identificar rotas "alternativas" usando softwares comerciais, tais como Word, Excel, Reference $M$ anager e $O$ rigin, buscando, assim, chegar a al go que possamos chamar de inovador no tratamento automatizado da informação.

\section{METODOLOGIA}

\section{E scolha da fonte de informação}

0 Institutefor Scientific I nformation (ISI) éa principal instância de indexação de artigos na área científica. A escolha desta fonte deve-se a uma série de fatores, além da facilidade de acesso via I nternet:

- o banco de dados de periódicos e respectivos artigos indexados do ISI são umaamostragem representativa da produção das comunidades científicaetécnica mundiais, tanto em termos de quantidade, em vista do grande número de periódicos que fazem parte deste banco, quanto qualitativamente, devido ao rigor de critérios de avaliação das revistas indexadas participantes deste grupo;

- neste banco de dados há tanto periódicos destinadosa trabalhos em ciência "pura", como também aqueles devotados aartigossobre "ciência aplicável" etecnologia;

- o banco de dados ISI tem impacto e repercussão na comunidade científica mundial, sendo normalmente usado parafinal idades múltiplas, desde a busca de artigos sobreum determinado assunto, atéa coletadedados para avaliação de pesquisadores e institutos.

\section{Escolha da área de aplicação}

Pessoas diferentes possuem visões distintas das organizações, a partir da base cultural e social em que estão inseridas. C onsiderando estepressuposto básico, pode-se inferir que o mesmo ocorre com o desenvolvimento de produtos em uma empresa, havendo então diferentes formas dese ver esta atividade, deacordo com as diferenças pessoais ea formação básicados envolvidos. 
A té bem pouco tempo, desenvolver produtos vinha sendo tratado de maneira isolada pelas diferentes áreas de conhecimento. A indahoje, profissionais de engenhariatendem a pensar o desenvolvimento deproduto como uma atividade específica composta de cál culos e teste. A Iternativamente, paradesignerse programadores visuais o produto aser desenvolvido éo resultado de estudos de conceitos; para administradores é al go mais abstrato, independente do conteúdo tecnológico e voltado para problemas organizacionais eestratégicos; enquanto que, para especialistas em qualidade, por seu turno, o produto é visto principalmente como aplicação de um instrumental específico destaárea (Rozenfeld et alii. 2000).

Tantas visões diferentes podem levar a problemas e até mesmo à ineficiência. $C$ ada visão parcial carrega consigo uma linguagem e determinados valores próprios, que dificultam a integração entre os profissionais pertencentes a cada uma dessas escolas. $\mathrm{N}$ o entanto, qualquer desenvolvimento de produto, por maior que seja a hegemonia de um determinado conteúdo tecnológico, deve ser um todo integrado que depende, para um adequado resultado final, dos conhecimentos de várias destas visões.

Enfrentar esta situação requer a construção de uma imagem única e integrada do processo de desenvolvimento de produto. U ma forma de conseguir isso é visual izar a real ização do desenvolvimento deprodutos por meio dealgum tipo de modelamento deste processo, agrupando-se então as fases envolvidas desde a proposição do conceito até o lançamento do novo produto e procurando realçar as conexões entretarefas e conhecimentos envolvidos nesse processo.

$\mathrm{N}$ este sentido, para auxiliar na modelagem do processo dedesenvolvimento deprodutos, um recurso útil éum Mapa de $C$ onhecimentos, cuja rotina de construção é umadenossas contribuições.

\section{MET O DO LO GIA ESPECIÍFICA}

Com a disposição de testar softwares alternativos para a análise bibliométrica, usamos o Word eo Excel, queestão disponíveis em praticamente qualquer computador pessoal, o R eference $M$ anager ( $R M$ ) e o 0 rigin $^{1}$, que também são muito populares. $C$ adaum delestem funções específicas: o W ord é usado para os textos; o Excel, para as planilhas; 00 rigin, para as figuras; o RM , para 0 gerenciamento de bases de dados e organização de referências.

${ }^{1}$ Word e Excel são utilitários da M icrosoft, R eference M anager é produzido pelaISI-R esearch Soft, eo $\mathrm{O}$ rigin éum produto da M icrocal.
FIGURA 1

$R$ elacionamento entre periódicos, descritores e pesquisadores

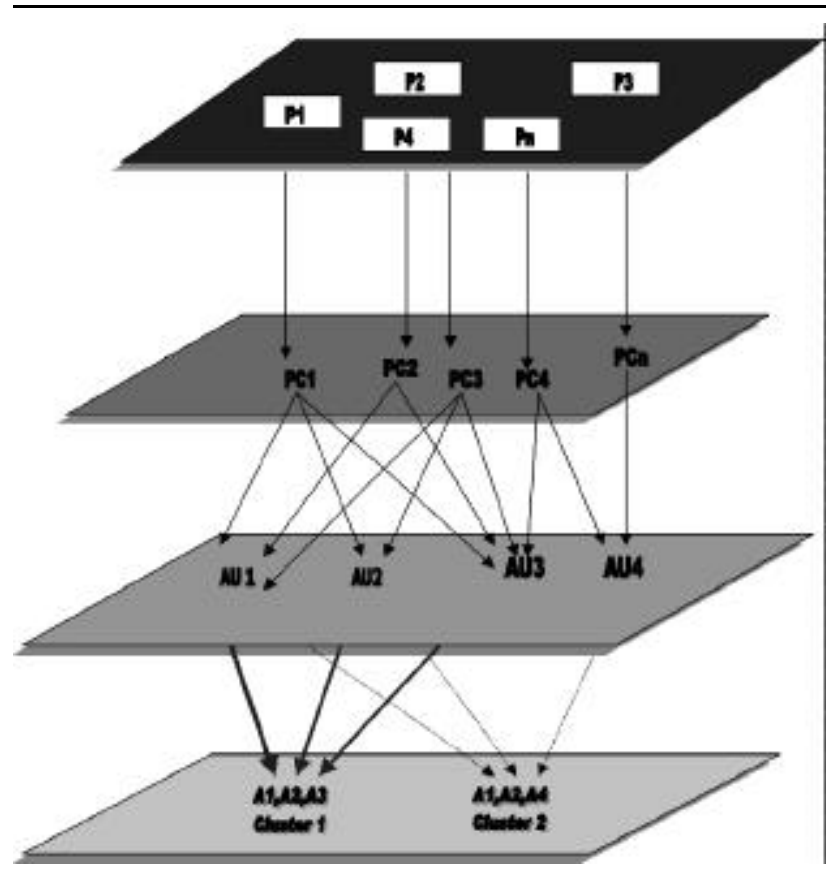

U sando o banco de dados do ISI , nas suasáreas de ciências e engenharias, que são os grandes grupos nos quais se encaixa o tema desenvolvimento de produtos, efazendo uso de métodos e ferramentas de tratamento automatizado da informação, levantamos um Mapa de C onhecimentos, como o esquematizado nafigural.

Com o Mapa, podemos detectar os relacionamentos eventualmente existentes entre as três entidades mencionadas ( periódicos, descritores/conhecimentos/ palavras-chave e autores), para a área de pesquisa em questão. A partir daí, será possível verificar seháclusters em torno deum determinado conhecimento, indicando quais são os pesquisadores eperiódicos mais relacionados com este e que, portanto, merecem maior atenção e acompanhamento sistemático.

Tomemos, como exemplo, um caso em que os conhecimentos (palavras-chave - PC) obtidos no A rtigo 1 são $P C 1$, PC 2 e PC 3 (temos, portanto, três relações: $P C 1 \ll P C 2 ; P C 1 \ll P C ~ 3$ ePC 2 $\ll P C ~ 3)$. Se as PC s obtidas no A rtigo 2 forem PC 1, PC 2, PC 3 (as mesmas do artigo anterior) e PC 4, teremos, portanto, seis relações: PC 1《PC 2; PC 1《PC 3; PC 1《PC 4; PC 2《PC 3; $P C 2 \ll P C 4$ ePC $3 \ll P C 4$. Procedendo-se de modo análogo para os demais artigos, obtém-se ao final umalistagem 
de palavras-chave ${ }^{2}$ (ou descritores) com um indicador de seus relacionamentos. A ssim, chamando de $n_{\mathrm{ij}} 0$ número de vezes que um certo par de palavras-chave $P C \mathrm{i} \ll P C \mathrm{j}$ aparece no cruzamento de quaisquer dois artigos, podemos representar os indicadores de relacionamento de uma forma matricial, que pode ter um preenchimento automatizado. Lembrando que $n_{i j}=$ $\mathrm{n}_{\mathrm{j} 1}$, teremos:

A lém deste relacionamento em nível horizontal (incluindo-se as matrizes "periódicosversusperiódicos" e "pesquisadores versus pesquisadores", conforme esquema na figura 1), étambém possível aquantificação de relacionamentos em níveis (horizontal «vertical), construindo-se matrizes com "periódicosversuspalavraschave", ou "pesquisadores versus palavras-chave", ou ainda "pesquisadores versusperiódicos".

A ssim, autilização deumaferramentaautomatizada para a construção destas inúmeras matrizes e de suas representações gráficas facilita a coleta de dados e sua análisecrítica.

\section{Escolha de descritores}

D escritores foram escolhidos a partir de uma lista elaborada por pesquisadores do $\mathrm{N}$ úcleo de $\mathrm{M}$ anufatura A vançada da U niversidade de São Paulo (EM U MA), campus São $C$ arlos, que, trabalhando naárea, conhecem as suas características e peculiaridades. Essa experiência resultou em uma primeiralista com as 75 palavras mais usadas na área, das quais, depois de busca na Web of Science, ficamos com 44 paraaanálisefinal. I sto porque, das 75 palavras pesquisadas, 31 resultaram em resposta nula, sendo então excluídas de nossa busca.

\section{Busca na base de dados Science C itation Index ISI}

A busca, restrita aos anos de 1995 a 2000, foi realizada por meio dos descritores mencionados anteriormente. A escolhadesse período em particular teveo objetivo de restringir o volume de informação sem perda de atualidade. Para nossa busca na Web, fixamos os parâmetros conforme a descrição que se segue:

Basepesquisada: ScienceC itation Index Expanded -1945present

Y ear selection: $20001999 \quad 1998 \quad 1997 \quad 1996 \quad 1995$

\footnotetext{
${ }^{2}$ N este trabalho, os termos "palavra-chave", "descritor" e "conhecimento" são tratados como sinônimos.
}

TABELA 1

R epresentação dos pares de PC 5

\begin{tabular}{llllll}
\hline & PC1 & PC2 & PC3 & PC 4 & etc \\
\hline PC 1 & - & $n 21$ & $n 31$ & $n 41$ & $\ldots$ \\
PC 2 & $n_{21}$ & - & $n 32$ & $n 42$ & $\ldots$ \\
PC 3 & $n_{31}$ & $n 32$ & - & $n 43$ & $\ldots$ \\
PC 4 & $n_{41}$ & $n 42$ & $n 43$ & - & $\ldots$ \\
etc & $\ldots$ & $\ldots$ & $\ldots$ & $\ldots$ & - \\
\hline
\end{tabular}

\section{SET LIMITSAN D SORT O PTIO N}

specific language: all

documenttype: articles

Sort results by: last date

\section{Procedimento de busca}

Primeiramente realizamos uma busca individual, com cada um dos descritores, obedecendo às seguintes etapas:

- Busca T ítulo: busca apenas no título dos artigos. N essa busca, obtivemos como respostas o número de artigos quecorrespondiam ao solicitado, sem a preocupação com a eventual existência de duplicatas.

- Busca Total: uma segunda busca, incluindo o títuloeo abstract, anotando-se o número de ocorrências sem a preocupação com duplicidade.

- B usca R efinada: fixando-se um descritor e utilizando o boleano A N D no cruzamento dessedescritor com cada um dos outros 43, pudemos verificar aexistênciadepares. $C$ ada descritor $X$ que, cruzado com o descritor $Y$, tenha resultado diferentedezero poderáentão ser considerado um "conhecimento" relevante. I sto significaque, em uma busca feita com esse par de descritores, o pesquisador efetivamente obterá resposta não-trivial da base de dados.

\section{A nálise dos dados}

D igitamostodos os números de ocorrências resultantes das buscas em uma planilhaExcel, montando uma matriz cujos elementos foram depois importados parao 0 rigin, onde produzimos a curva conhecidacomo "J invertido". Esse procedimento foi repetido paraas duas buscas, isto é, tanto nos dados obtidos na Busca Título como naqueles obtidos na Busca Total. 0 procedimento érepetido por completeza, para a verificação de cada uma das leis bibliométricas. 
D epois de anal isadas as palavras, passamos a estudar as relações existentes entre os autores, verificando a formação de clusters, isto é, quem trabalha com quem na área de desenvolvimento de produto. Finalizamos nossa pesquisa analisando quais são os periódicos de maior relevância naárea.

Em seguida, apresentamos fluxogramas representativos dos procedimentos empregados em buscas e análise envolvendo Lista de Periódicos, Listas de D escritorese C lusters de A utores (figuras 2, 3 e 4 ).

FIGURA 2

Fluxograma para lista de periódicos

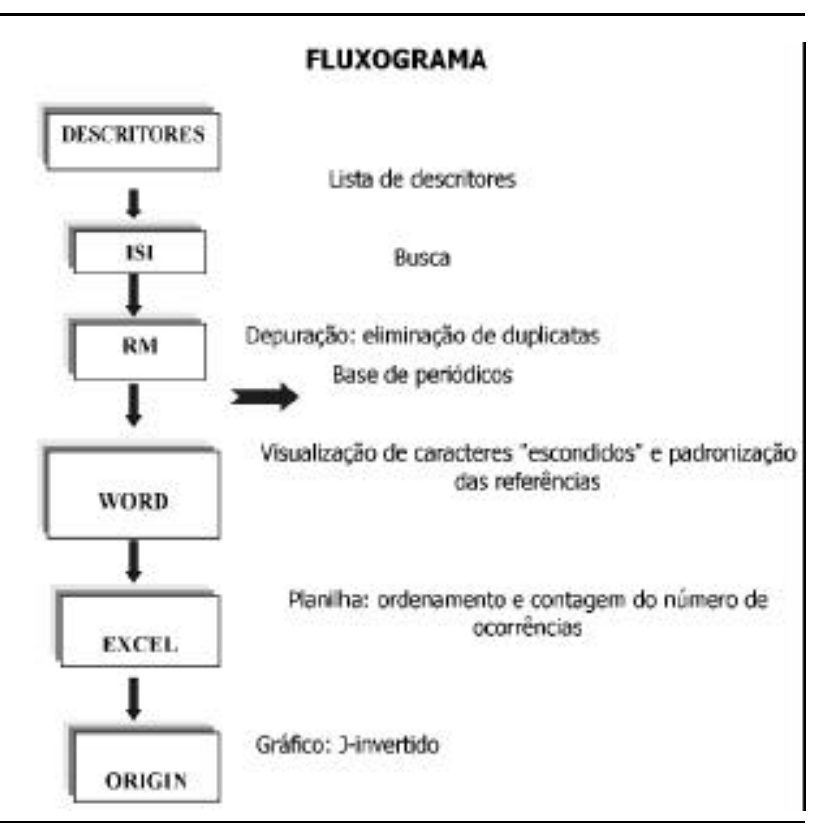

FIGURA 3

Fluxograma para lista de descritores

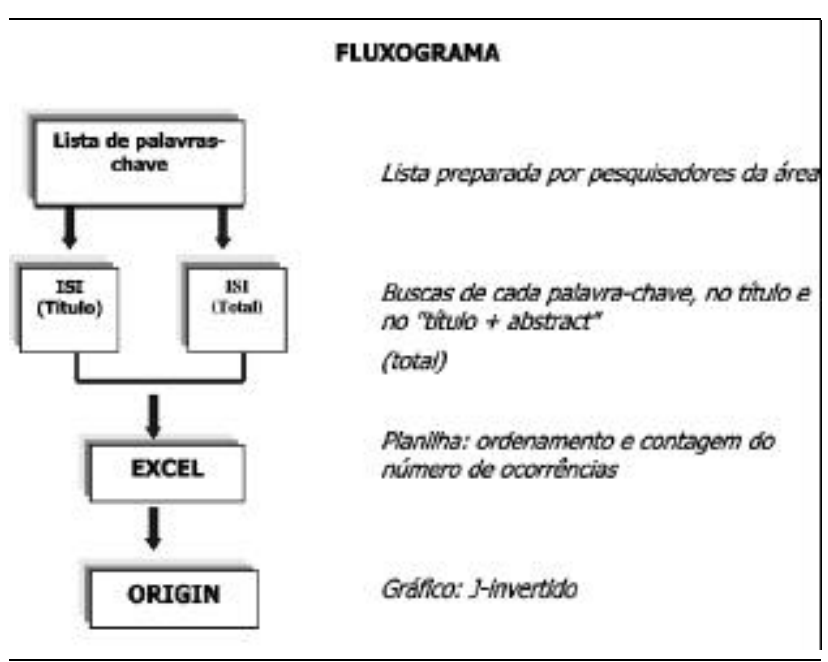

FIGURA 4

\section{Fluxograma para cluster de autores}

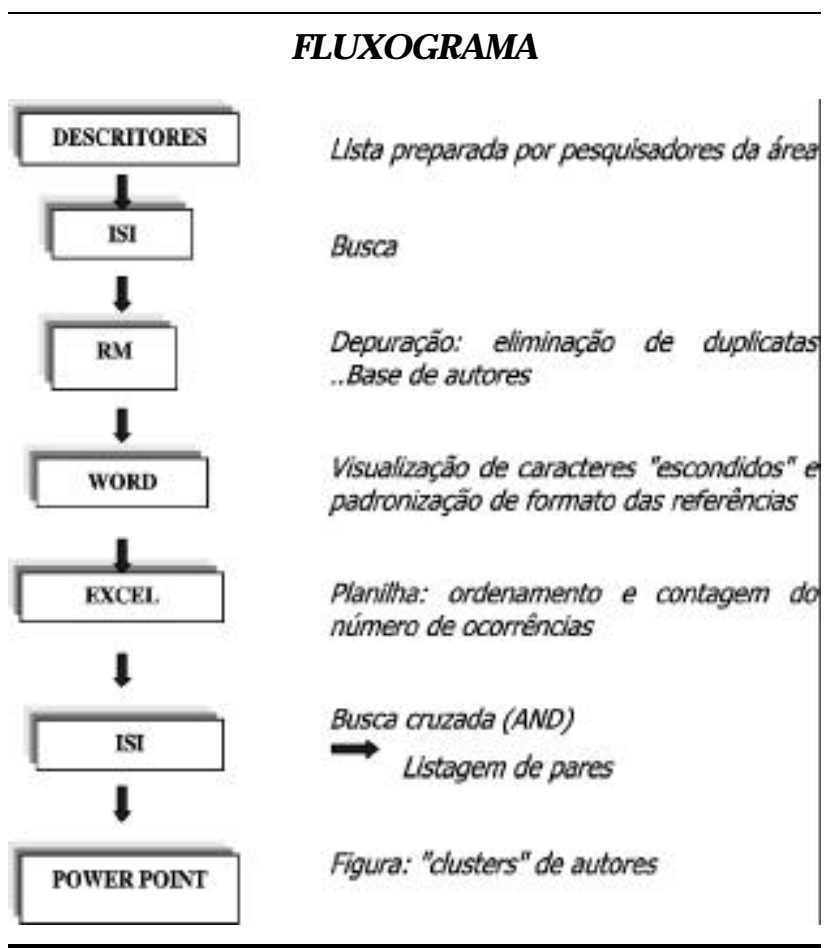

R esultados - procedimento detalhado

\section{D escritores}

A partir dalistagem dedescritoresinicialmenteproposta, foram escolhidos os "não-triviais", que formam a base do trabalho. 0 s descritores são mostradosnatabela2, a seguir. Para cada um deles, realizamos dois tipos de busca individual, uma procurando pelo descritor apenas no título e a outra incluindo título eabstract, conforme discutimos anteriormente.

A s respostas obtidas para cada um dos descritores são resumidas na tabela 2 . $\mathrm{N}$ a figura 5 , a seguir, temos 0 gráfico J-invertido para o número de ocorrências dos descritoresnostítulos. 0 número deordem do descritor no eixo horizontal foi atribuído segundo a freqüência das respostas. A figura 6 mostrao gráfico J-invertido para o número de ocorrências dos descritores no total, isto é, títulos eabstract. C omo nafigura anterior, o número de ordem do descritor foi atribuído de acordo com afreqüência das respostas.

Segundo a representação daL ei deZipf pelo modelo de "N úcleo eD ispersão" (Potter,1981; R ostaing, 1996), a porção mais significativa dos descritores estaria concentradanos $20 \%$ mais freqüentes, como mostramos nas figuras 6 e 7, a seguir. A alternativa proposta por

Ci. Inf., Brasília, v. 31, n. 3, p. 66-76, set./dez. 2002 
TABELA 2

R espostas das buscas dos 36 descritores restritas ao título e total incluindo (título + abstract). $0 \mathrm{~s}$ descritores estão listados em ordem alfabética.

\begin{tabular}{|c|c|c|}
\hline \multirow[b]{2}{*}{ D escritor } & \multicolumn{2}{|c|}{$\mathrm{N}$ úmero de $\mathrm{O}$ corrências } \\
\hline & Título & Total \\
\hline Benchmarking & 281 & 579 \\
\hline Bill of material & 2 & 16 \\
\hline Business process & 129 & 371 \\
\hline Change management & 16 & 112 \\
\hline Competitive strategy & 18 & 65 \\
\hline Computer aided design & 209 & 1179 \\
\hline Computer aided manufacturing & 13 & 105 \\
\hline Computer aided process & 50 & 166 \\
\hline Concurrent engeneering & 215 & 577 \\
\hline D esign of experiments & 106 & 509 \\
\hline Enterprise resource planning & 9 & 28 \\
\hline F ast prototyping & 6 & 24 \\
\hline FMEA & 12 & 51 \\
\hline FTA & 15 & 135 \\
\hline$G D \& T$ & 7 & 15 \\
\hline Lean products & 0 & 1 \\
\hline Modeling products & 1 & 1 \\
\hline Modular products & 5 & 11 \\
\hline Pareto & 144 & 599 \\
\hline PDM & 100 & 248 \\
\hline PokaYoke & 0 & 2 \\
\hline Product development times & 1 & 4 \\
\hline Product data management & 23 & 52 \\
\hline Product development & 366 & 1197 \\
\hline Product management & 176 & 549 \\
\hline Prototyping virtual & 0 & 3 \\
\hline QFD & 0 & 8 \\
\hline Q uality function deployment & 30 & 98 \\
\hline Q uality system & 60 & 248 \\
\hline Supply chain management & 45 & 113 \\
\hline Taguchi & 97 & 427 \\
\hline Theory of constraints & 19 & 57 \\
\hline Value analysis & 43 & 170 \\
\hline Virtual mock-up & 0 & 1 \\
\hline Virtual time & 8 & 30 \\
\hline Workgroup computing & 2 & 7 \\
\hline
\end{tabular}

outros autores (R ostaing, 1993, R ostaing, 1996; F aria, 1995; Q uonian, 1997) separa a curva em três zonas para casos como este, em que o elemento bibliométrico pertence aum vocabulário controlado.

Entretanto, não havendo consenso quanto às porcentagens de cada zona e tendo em conta que é pequeno o número dedescritores, decidimos não nos deter neste aspecto.

\section{Busca com booleano A N D e construção da matriz D}

C omo o número dedescritores com resposta não-nulaé relativamente pequeno, vamos incluir todos os $36 \mathrm{em}$ nossa amostragem. Seo número de descritoresfosse uma
FIGURA 5

G ráfico J-invertido para o número de ocorrências dos descritoresnostítulos. 0 número de ordem do descritor no ei xo horizontal foi atribuído segundo a freqüência das respostas

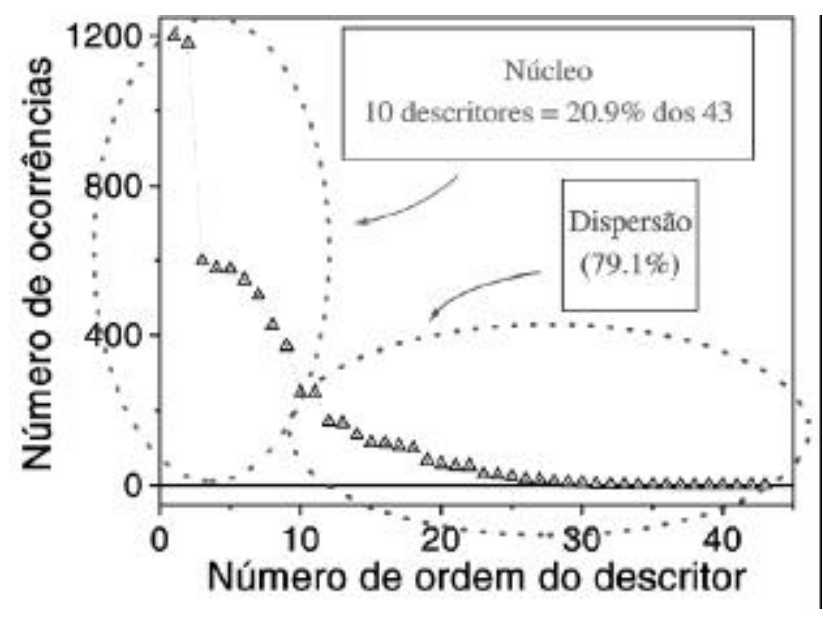

FIGURA 6

G ráfico J-invertido para o número de ocorrências dos descritores no total, isto é, títulos + abstract. 0 número de ordem do descritor foi atribuído segundo a freqüência das respostas

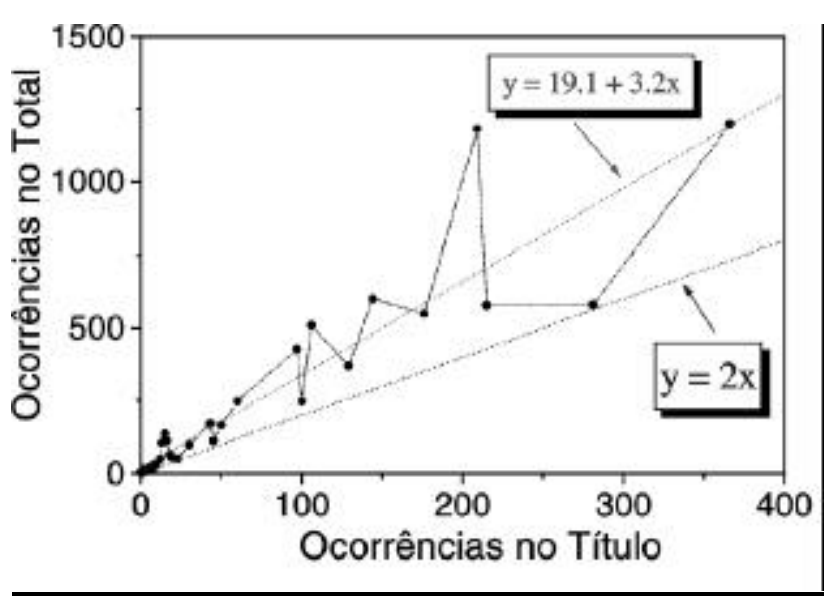

ou duas ordens degrandeza maior, seriasensato desprezar os 20\% correspondentes àregião de ruído. Fixada nossa amostragem, o próximo passo foi o cruzamento entre eles. Para isso, realizamos uma nova busca no ISI, mantendo os mesmos parâmetros anteriores eusando o boleano AN D . F ixamos um dosdescritorese, realizando a busca com cada um dos outros, encontramos as correlações entre 35 pares de descritores. Em seguida, fixamos um segundo descritor e, realizando a buscacom A N D , determinamos outras 34 correlações entre pares, 
e assim sucessivamente até chegarmos ao par formado pelo penúltimo e o último dosdescritores.

U ma maneira elegante e concisa de catalogar os resultados dessas buscas como boleano A N D é através deumamatriz ( D ), cujos elementos diagonais, $D_{i i}$, são os resultados das buscas individuais de cada descritor, enquanto os não-diagonais, $D_{i j}$, representam as correlações entre dois descritores, conformeas respostas obtidas com o boleano A N D. A ssim, quanto maior o val or do elemento diagonal, mais freqüenteé o descritor no universo pesquisado. $D$ amesmaforma, quanto maior o valor do elemento não-diagonal, maior a correlação entre os dois descritoresque ele representa. 0 bviamente a matriz $\mathbf{D}$ tem simetria especular em relação à diagonal $\left(D_{i j}=D_{j i j}\right)$, razão pela qual construímos apenas a sua metadesuperior $\left(\mathrm{j}^{3} \mathrm{i}\right)$.

O A pêndice 1 apresenta a matriz $\mathbf{D}^{\text {Tit }}$, construídacom os resultados de buscas nos títulos, no formato de uma planilhaExcel. Também no A pêndice 1, temos a matriz D ${ }^{\text {tot, }}$ com os resultados de buscas no total (títulos e abstract), igual menteno formato Excel.

\section{C lusters de autores}

U m dos objetivos deste trabalho foi o estabelecimento deum procedimento sistemático paradetectar aformação de clusters (grupos) de pesquisadores que trabal ham no mesmo assunto e publicam como co-autores. A lém de determinar a composição desses agrupamentos, deseja-se detectar as eventuais interações entre diferentesclusters.

A estratégia empregada para o estudo que levou ao estabelecimento da rotina de monitoramento baseia-se primordialmente na determinação dos autores mais importantes da área, por meio da contagem do número depublicações queincluam pelo menos um dos principais descritores dessa mesmaárea, seguindo o procedimento deverificação no título eno abstract. Em seguida, verificamse quais são os co-autores de cada autor em particular, delineando assim os clusters e sua eventual correlação.

Paratanto, começamos pelacriação deumabasededados, consolidando por meio do RM (R eferenceM anager) os resultados das buscas no ISI. U tilizando-nos das ferramentas do utilitário, depuramos a base, eliminando todas as ocorrências duplicadas, ou seja, artigos idênticos que resultaram das buscas com diferentes descritores. Essa base de dados depuradatornou-seo universo para os tratamentos de informação que se seguiram.

\section{FIGURA 7}

\section{G ráfico mostrando a correlação entre título e total}

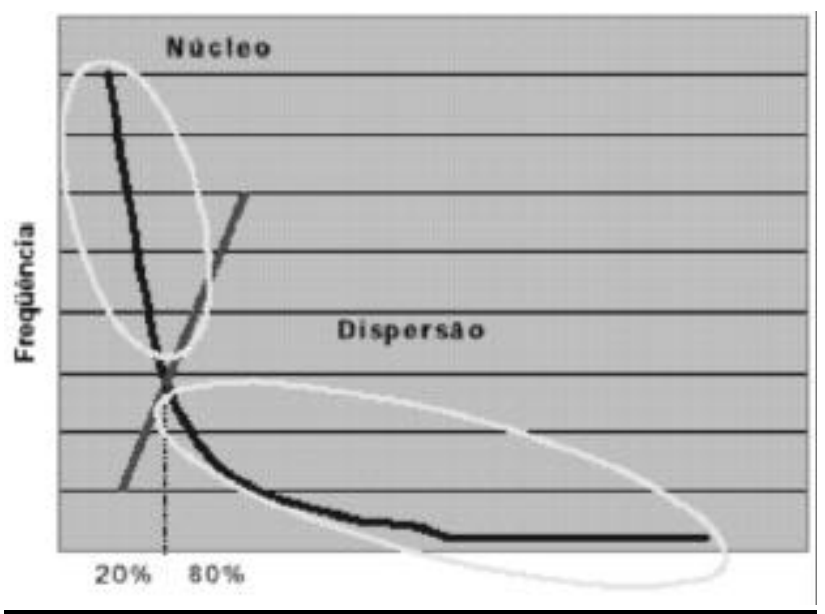

Primeiramente, usando a base depurada, construímos uma base-espelho em que, utilizando-nos do filtro adequado, importamos apenas o campo A U TO R (AU ) decadareferência. $O$ filtro pode ser escolhido dentreos existentes no RM , ou editado, ou mesmo criado. U ma seqüência esquemática de edição e uso do filtro no RM pode ser vista na figura 7. A base-espelho foi então exportada para o Word ( $\mathrm{N}$ otePad seria igualmente adequado), paraa edição de caracteres espúrios comenters, caracteres invisíveis, eventuais símbolos decampos não importados etc. Tão importantequanto essa depuração decaracteres foi garantir quetodos os autores formassem uma colunaúnica, paraser posteriormente ordenada, de modo que ocorrências repetidas de um mesmo autor aparecessem em seqüência, facilitando a contagem das ocorrências de cada autor. Resolve-se assim, automaticamente, a questão fundamental de quetodos os autores de um artigo devem ter tratamento idêntico nesta abordagem, independentemente da ordem em que apareçam na lista de autores de cada artigo.

A bre-se então o Excel para a importação do arquivo derivado da base-espelho, que, devidamente depurado, foi montado de modo que todos os autores com sobrenomes iniciados por determinada letraocupassem uma única coluna, o que facilita o ordenamento alfabético. D eum total de6.924 autores da base-espelho, selecionamos os mais freqüentes ( $M$ F s - todos com duas ou mais citações) paraumanovabuscano ISI , objetivando assim detectar os cl usters que desejamos identificar.

O bservando os Mapasdafigura 8, concluímos que não existe, pelo menos em nossa amostragem retirada do ISI, interação entre os diversos clusters. 0 que temos são 
clustersisolados, isto é, um autor principal com al guns colaboradores mais freqüentes, trabalhando de forma estanque. Pesquisadores daáreatendem a reconhecer esse "isolamento", que parece ser devido à multidisciplinaridade daárea. A formação de "nichos" pareceser um procedimento comum na área monitorada, o que confirma como confiável o diagnóstico decorrente do procedimento utilizado.

\section{C onstrução do J-invertido para os periódicos}

U m bom exemplo decomo informações relevantes podem ser obtidas como decorrência de um processo específico de monitoramento de informação é mostrado aqui por meio do gráfico do tipo J-invertido para os periódicos. Trata-se de uma figura que realça os periódicos mais importantes daárea, cuja construção foi feita a partir da mesma base de dados depurada que utilizamos para produzir os M apasdeclusters de autores.

$\mathrm{N}$ afigura9, temos o gráfico J-invertido paraos periódicos, indicando dois deles como exemplos: C omputers and I ndustrial E ngeneering, com 217 ocorrências, eVehicle System $D$ ynamics, com oito. A inserção da figuramostra um detalhe da região dos periódicos com menor incidência, em que se notam degraus de altura unitária que vão se alargando até o patamar de uma só citação, queéo maislargo, ou seja, o mais freqüente.

\section{ONSIDERAÇ ÕES FIN AIS}

$\mathrm{N}$ este artigo, descrevemos um estudo sistemático de monitoramento da informação, focalizado na área de desenvolvimento deprodutos. 0 estudo baseou-se em resultados obtidos na base ISI - Web of Science e demonstra a utilidade potencial do método para a obtenção de diagnósticos confiáveis.

Executando a sistemática proposta, obtivemos a relação dos periódicos mais usados na área, incluindo a freqüência da citação. 0 btivemos também a lista de descritores que devem ser empregados em buscas de monitoração daárea, de modo a minimizar o trabalho de busca sem perda de eficácia. M ostramos também como se pode construir os mapas de autores da área, determinando os clusters de correlação. Sua correta interpretação possibilita uma leitura dinâmica do movimento daárea.

A utilização desoftwares como Excel, W ord, R eference $M$ anager e $O$ rigin, alternativamente aos tradicionalmenteempregados paraessefim, deu-nos maior liberdade detrabal ho, no quediz respeito ao acesso a ferramentas
FIGURA 8

Mapa de autores, mostrando a interação entre os mesmos para a formação declusterse, ao mesmo tempo, a completa independência entre diferentesclusters

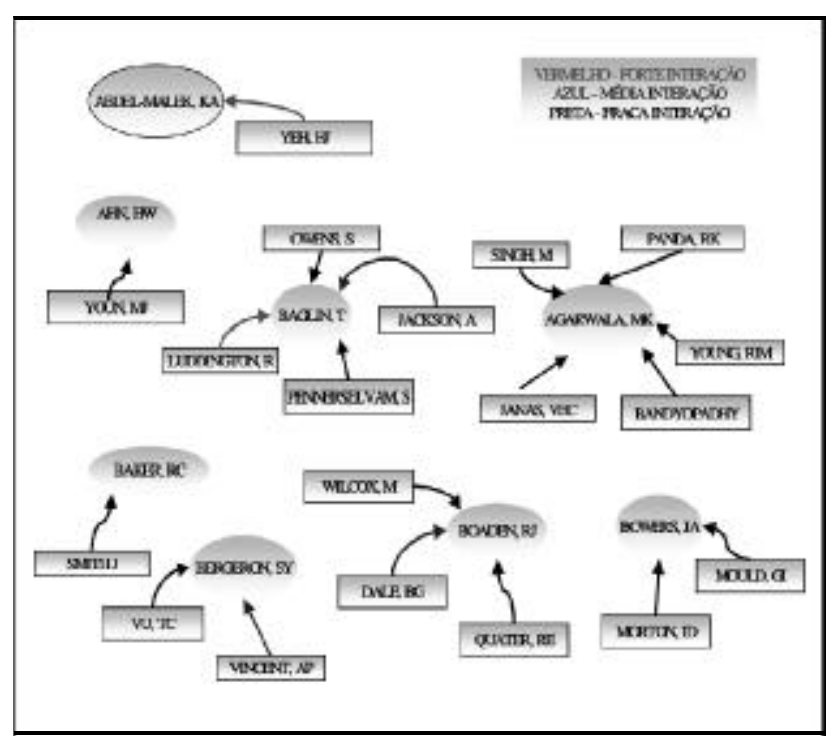

Forte interação - $\mathbf{3}$ ou mais trabalhos publicados juntos

M édia interação - $\mathbf{2}$ trabalhos publicados juntos

Fraca interação - apenas 1 trabalho publicado juntos

\section{FIGURA 9}

J-invertido dosperiódicos, realçando o maisfreqüente, bem como um outro de baixa ocorrência. 0 s degraus mostrados na inserção são comentados no texto

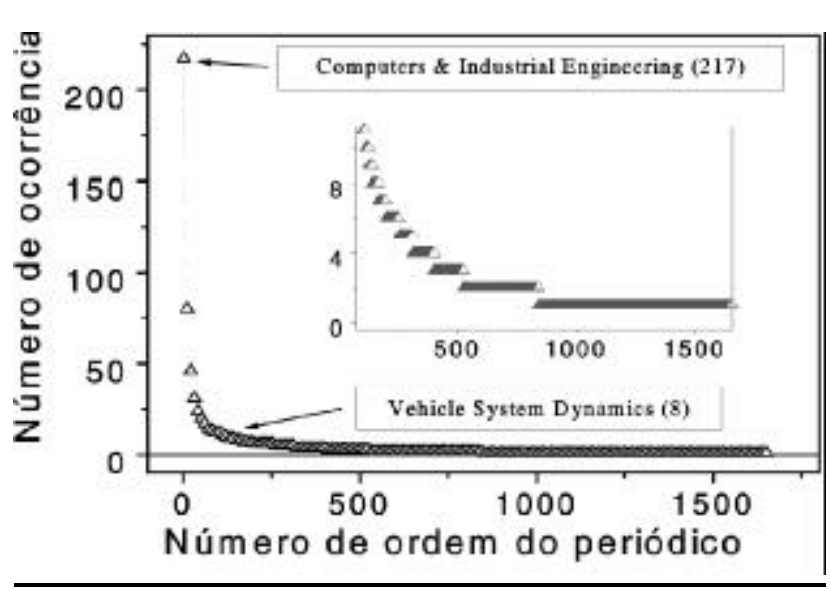

alternativas e de custos muito inferiores às raras ferramentas comerciais de monitoramento existentes. A ssim, esperamos que outros pesquisadores dispostos a monitorar suas áreas deatuação possam interessar-sepor testar estenosso procedimento.

A rtigo aceito para publicação em 24-04-2002 


\section{Lúcia C unha 0 rtiz / W ilson A ires 0 rtiz / Sergio L uis da Silva}

\section{REFERÊNCIAS}

FA RIA , L. I. L. I nformação tecnológica eseleção de materiais: estudo de caso sobre pastilha de freios automotivo. 1995. 192 f. D issertação (M estrado) - D epartamento deEngenharia de M ateriais, U niversidade Federal de São C arlos, São C arlos, 1995.

PO TTER, W. G. Lotka's Law revised. Library Trends, v. 30, n. 1, Summer 1981.

Q U O N I A , L uc et al. Bibliometri a law used for information retrieval. In: CON FEREN CE OF THE IN TERN ATION AL SO CIETY FOR SCIEN TO METRICSAN D IN FO RMETRICS, 6th, 1998. Proceedings.. [s. I.] : H ebrow U niversity of J erusalem., 1998.

RO STA IN G, H . La bibliométrie et sestechniques. Toulouse: Sciences de la societé, 1996.

Veille technologique et bibliométrie: concepts, outils, appplication. 1990. 353 f. Tese (D ouorado) - Faculté des Sciences et Techniques de Saint Jérôme, U niversité de $D$ etroit et des Sciences dÁ ix-M arseille, M arseille, 1993.

RO ZEN FELD , H . at al. D evelopment of a concurrente engineering education environment. I nternational J ournal of $C$ omputer I ntegrated M anufacturing, v. 13, n. 6, p. 475-482, N ov./D ec. 2000.

SO TOLONGO-AGUILAR, G .; SUARÉZ-BALSEIRO, C . ; GU ZM ÁN - SÁ N C H EZ, M . M odular bibliométrics information system with proprietary software. [s. I. : s. n.], 1999.

TO LED O , J. C. G estão da mudança da qualidade de produto. 1993. Tese (D outorado) - Escola Politécnica da U SP, São Paulo, 1993. 
Ferramentas alternativas para monitoramento e mapeamento automatizado do conhecimento

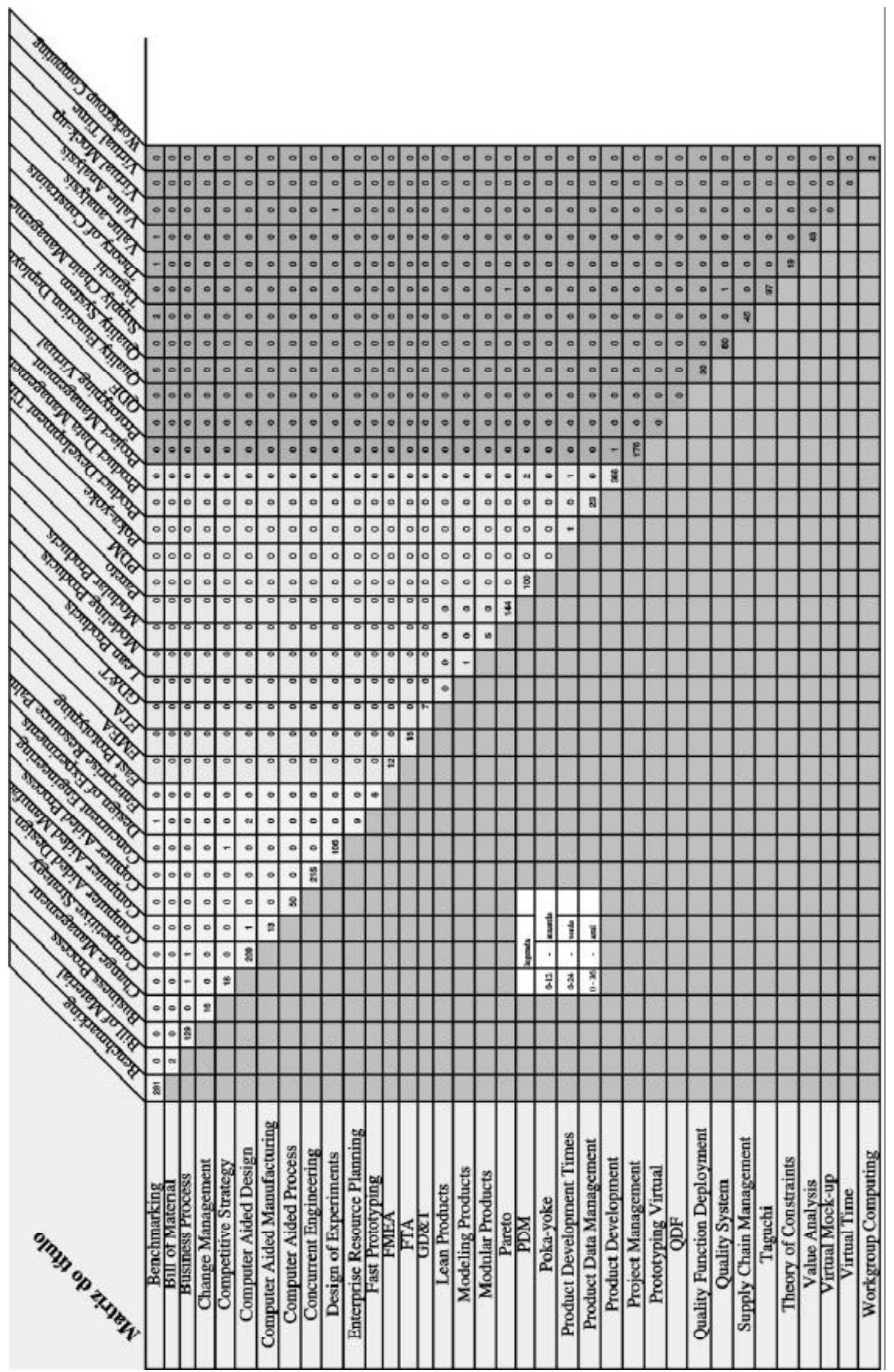

Ci. Inf., Brasília, v. 31, n. 3, p. 66-76, set./dez. 2002 
Lúcia C unha 0 rtiz / W ilson A ires 0 rtiz / Sergio L uis da Silva

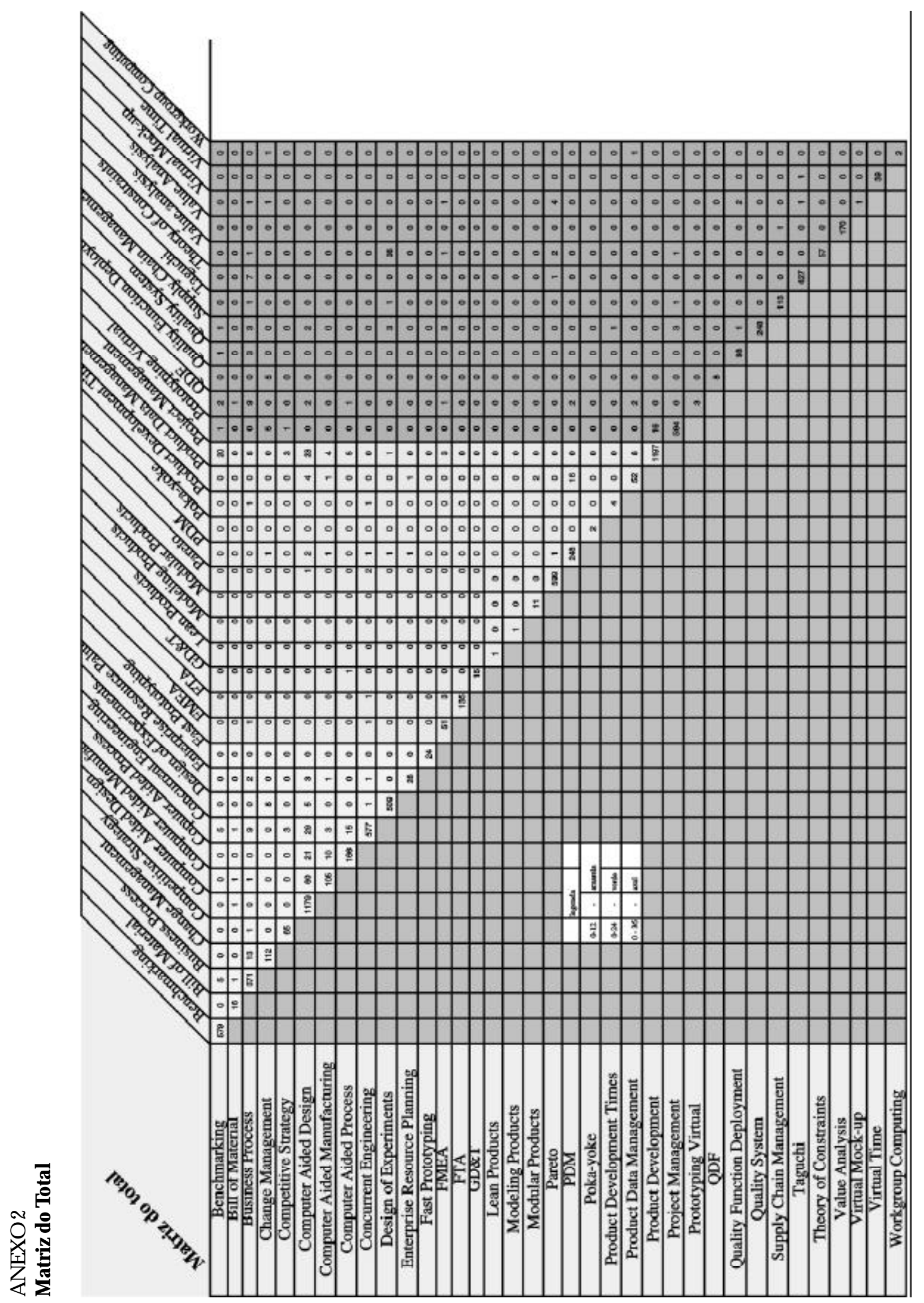

